\title{
Can absence seizures be predicted by vigilance states?: Advanced analysis of sleep-wake states and spike-wave discharges' occurrence in rats
}

\author{
Magdalena K. Smyk ${ }^{\text {a,b }}$, Ilya V. Sysoev ${ }^{\text {c,d, }}$, Marina V. Sysoeva ${ }^{\text {e,d }}$, \\ Gilles van Luijtelaar ${ }^{\mathrm{f}}$, Wilhelmus H. Drinkenburg ${ }^{\mathrm{g}}$ \\ a Malopolska Centre of Biotechnology, Jagiellonian University in Krakow, Poland \\ ${ }^{\mathrm{b}}$ Department of Neurophysiology and Chronobiology, Institute of Zoology and Biomedical Research, Jagiellonian University in Krakow, Poland \\ c Saratov State University, Saratov, Russia \\ d Saratov Branch of Kotel'nikov Institute of Radio Engineering and Electronics, Saratov, Russia \\ e Yuri Gagarin State Technical University of Saratov, Saratov, Russia \\ ${ }^{\mathrm{f}}$ Donders Centre of Cognition, Radboud University, Nijmegen, the Netherlands \\ ${ }^{g}$ Department of Neuroscience, Janssen Research and Development, Janssen Pharmaceutica N.V., Beerse, Belgium
}

\section{A R T I C L E I N F O}

\section{Article history:}

Received 11 January 2019

Revised 27 March 2019

Accepted 8 April 2019

Available online $\mathrm{xxxx}$

\section{Keywords:}

Spike-wave discharges

Sleep-wake states

WAG/Rij rats

Predictability

Conditional Shannon entropy

Absence epilepsy

\begin{abstract}
A B S T R A C T
Spike-wave discharges (SWDs) are the main manifestation of absence epilepsy. Their occurrence is dependent on the behavioral state, and they preferentially occur during unstable vigilance periods. The present study investigated whether the occurrence of SWDs can be predicted by the preceding behavioral state and whether this relationship is different between the light and the dark phases of the 24-h day.

Twenty-four-hour (12:12 light/dark phases) electroencephalographic (EEG) recordings of 12 Wistar Albino Glaxo, originally bred in Rijswijk (WAG/Rij) rats, a well-known genetic model of absence epilepsy, were analyzed and transformed into sequences of 2-s length intervals of the following 6 possible states: active wakefulness (AW), passive wakefulness (PW), deep slow-wave sleep (DSWS), light slow-wave sleep (LSWS), rapid eye movement (REM) sleep, and SWDs, given discrete series of categorical data. Probabilities of all transitions between states and Shannon entropy of transitions were calculated for the light and dark phases separately and statistically analyzed.

Common differences between the light and the dark phases were found regarding the time spent in AW, LSWS DSWS, and SWDs. The most probable transitions were that AW was preceded and followed by PW and vice versa regardless of the phase of the photoperiod. A similar relationship was found for light and deep slow-wave sleep. The most probable transitions to and from SWDs were AW and LSWS, respectively, with these transition likelihoods being consistent across both circadian phases. The second most probable transitions around SWDs appeared more variable between light and dark. During the light phase, SWDs occurred around PW and participated exclusively in sleep initiation; in the dark phase, SWDs were seen on both, ascending and descending steps towards and from sleep. Conditional Shannon entropy showed that AW and DSWS are the most predictable events, while the possible prediction horizon of SWDs is not larger than $4 \mathrm{~s}$ and despite the higher occurrence of SWDs in the dark phase, did not differ between phases.

It can be concluded that although SWDs show a stable, strong circadian rhythm with a peak in number during the dark phase, their occurrence cannot be reliably predicted by the preceding behavioral state, except at a very short time base.
\end{abstract}

(c) 2019 Elsevier Inc. All rights reserved.

\footnotetext{
* Corresponding author at: Saratov State University, Saratov, Russia.

E-mail addresses: magdalena.smyk@gmail.com (M.K. Smyk), ivssci@gmail.com

(I.V.Sysoev), bobrichek@mail.ru (M.V.Sysoeva),g.vanluijtelaar@donders.ru.nl (G. van Luijtelaar),WDRINKEN@its.jnj.com (W.H. Drinkenburg).
}

\section{Introduction}

Bilateral, symmetrical, and synchronous spike-wave discharges (SWDs) are the electroencephalographic (EEG) hallmarks of absence epilepsy syndromes belonging to the group of genetic generalized epilepsies (GGE) [1,2]. Clinically, SWDs are accompanied by sudden arrest of ongoing activity, reduced awareness, and responsiveness lasting on average of a few seconds [3]. Initiated by a cortical focus, this particular epileptic 
activity is maintained by the cortico-thalamo-cortical network that is also engaged in vigilance control and manifestation of sleep-wake states $[4,5]$. Indeed, a strong, well-documented relationship between SWDs and the vigilance level exists. Absences are most numerous during stages I and II of slow-wave sleep, and least frequent during REM (rapid eye movement) sleep [6]. When emerging from wakefulness, they usually occur during inactivity and drowsiness, less often during high arousal states such as physical and engaging mental activity $[7,8]$. Such dependence determines a nonrandom distribution of absences across the circadian cycle $[9,10]$.

Knowledge about when seizures are occurring possesses large clinical utility. This principle is applied when seizure provocation techniques are used to facilitate the diagnosis, and sleep deprivation, a major factor precipitating seizures, is commonly used as a diagnostic tool [11,12]. Other examples of factors that influence the presence and timing of seizures are age of the patients and the localization of the focus, both appeared to be good predictors for the occurrence of epileptic discharges [13]. The main goal of epilepsy management is complete seizure control, and given that pharmacological treatment is ineffective in about $30 \%$ of patients, novel approaches based on seizures prediction are currently developed [14,15].

The WAG/Rij strain of rats is a well-known, extensively validated animal model of childhood absence epilepsy (CAE), one of the GGE syndromes [16]. A number of factors influencing the probability of seizure occurrence in this strain have already been explored, such as the age of the rats [17,18], the circadian timing system [19,20], and sex hormones [21]. Mathematical approaches were also used to describe the timing of seizures' occurrence such as time series analysis of a binary SWDs' signal constructed from long-term EEG recordings [22], the hazard rate, the conditional probability that a seizure starts in the time interval [t,t $\Delta$ ], given that no seizure has started in the time interval [0, t] [23], and whether principles of intermittency (irregular alternation of phases of apparently periodic and chaotic dynamics) can be demonstrated $[24,25]$. Other approaches that were used to predict the SWDs' occurrence were based on time-frequency analyses of cortical and thalamic preictal EEG [26], multichannel network analysis of preictal EEG correlates [27-30], and multichannel synchronization analysis [15]. Finally, considering that SWDs in WAG/Rij rats are strongly influenced by vigilance, analysis of the level of vigilance, or more precise, the different sleep-wake states precipitating the occurrence of SWDs was used [31]. Passive wakefulness (PW) and light slow-wave sleep (LSWS) were found to be favorable, while active wakefulness (AW), deep slow-wave sleep (DSWS), and REM sleep were found to be unfavorable for the occurrence of SWD [32].

The goal of the present study was to assess whether the occurrence of SWDs can be reliably predicted based on the occurrence of sleepwake states as assessed during a 24-h period. Conditional probabilities combined with Shannon entropy were used considering interdependencies between the time series created from individual hypnograms. Considering the presence of a circadian rhythm of SWDs and the fact that the majority of the previous studies were restricted to either one phase of the photoperiod or single hours only, a distinction with regard to the light and the dark phases, respectively, has been made.

\section{Material and methods}

\subsection{Animals}

Twelve adult, male WAG/Rij rats (Harlan, the Netherlands) weighting $315 \mathrm{~g} \pm 12 \mathrm{~g}$ (about eight months of age at time of surgery) were used. Animals were singly-housed in individually ventilated Plexiglas cages $(25 \times 22 \times 18 \mathrm{~cm})$ in a sound-attenuated room and controlled environmental conditions (temperature: $22 \pm 2{ }^{\circ} \mathrm{C}$; humidity: $60 \%$; $12: 12$ light-dark cycle; white lights on at 05:00 am, off at 17:00 pm; light-dark switch via a dusk-dawn transition of $10 \mathrm{~min}$; light intensity: $\approx 100 \mathrm{~lx}$ ) throughout the experiment, as well as during the recovery period after the surgery. Standard laboratory chow (SAFE
A04, Augy, France) and tap water were available ad libitum. All protocols have been carried out in accordance with the guidelines of the Association for Assessment and Accreditation of Laboratory Animal Care International (AAALAC), and of the European Communities Council Directive of 24 November 1986 (86/609/EEC) and were approved by the Janssen Pharmaceutica Ethical Committee. Every effort was made to minimize animal use and disturbances in animal well-being. In case animals could not be reused, they were euthanized at the end of the study by a conventional rodent $\mathrm{CO}_{2}$ euthanasia procedure.

\subsection{Surgery}

Preceding surgery, the analgesic piritramide (Dipidolor; Janssen Pharmaceutica NV, Belgium) was administered. Under deep isoflurane inhalation anesthesia (i.e., a mixture of $\mathrm{O}_{2}, \mathrm{~N}_{2} \mathrm{O}$, and $2 \%$ isoflurane), seven stainless steel fixing screws (diameter: $1 \mathrm{~mm}$ ) were inserted bilaterally in the left and right hemispheres, respectively, along the anteroposterior axes in the following coordinates: anterior-posterior (AP): $2 \mathrm{~mm}$, lateral (L): $\pm 2 \mathrm{~mm}$; AP: $-2 \mathrm{~mm}$, L: $\pm 2 \mathrm{~mm}$; and AP: $-6.6 \mathrm{~mm}$, $\mathrm{L}: \pm 2 \mathrm{~mm}$ from Bregma; and referenced to the same ground electrode place midline above of the cerebellum. The incisor bar was $-5 \mathrm{~mm}$ under the center of the ear bar [33]. In addition, stainless steel wire electromyography (EMG) electrodes were placed in the muscles of the neck (7N51465T5TLT, 51/46 Teflon Bilaney, Germany). Electrodes were connected to a pin (Future Electronics: 0672-2-15-15-30-27-10-0) with a small insert (track pins; Dataflex: TRP-1558-0000) and were fit into a 10-holes connector, then the whole assembly was fixed with dental cement to the cranium. Rats were allowed to recover at least 10 days after surgery.

\subsection{EEG and EMG recordings}

Rats, while staying in their home cages, were placed in the soundattenuated recording boxes and connected by a cable to rotating swivels, allowing free movement during recording procedures. Rats were allowed to habituate to the recording set-up for $24 \mathrm{~h}$ preceding the experiment. Continuous EEG and EMG signals were acquired at a 2-kHz sample rate with an input range of $\pm 500 \mathrm{mV}$ through a Biosemi ActiveTwo system (Biosemi, Amsterdam, the Netherlands) referenced to the Common Mode Subtraction-Drift Right Leg (CMS-DRL) ground (common mode reference for online data acquisition and impedance measures, which is a feedback loop driving the average potential across the montage close to the amplifier zero). The signals were amplified and analogue bandpass filtered between 1 and $100 \mathrm{~Hz}$ and were digitized with 24-bit resolution.

\subsection{Analysis and classification}

Twenty-four-hour EEG and EMG recordings were analyzed. The following five vigilance states: AW, PW, LSWS, DSWS, and REM sleep, as well as a number of transitions between them, were identified automatically for 2-s epochs based on EEG, EMG, and body movements (passive infrared, PIR detection) following validated criteria [34] (Fig. 1). The duration of the epoch length was chosen to obtain a desired level of frequency resolution and, at the same time, to avoid nonstationarity segments [35]. An epoch length of $2 \mathrm{~s}$ was found to satisfy both criteria: stationarity and stability for power analysis, which is crucial in systems using power spectra and their ratios for sleep-wake determination [36]. A 2-s epoch is short enough to prevent the statistical parameters form changing during the epoch (it provides the smallest possible deviance in beginning-ending of stages) and to follow the rat sleep-wake dynamics [34,36]. Moreover, this particular epoch duration was also chosen with regard to the duration of SWDs (they have a variable length from 1 to ca $10-15$ s), which, together with their occurrence across $24 \mathrm{~h}$, was scored automatically based on the EEG signal from a frontal electrode according to well-established criteria: spike-wave complexes 

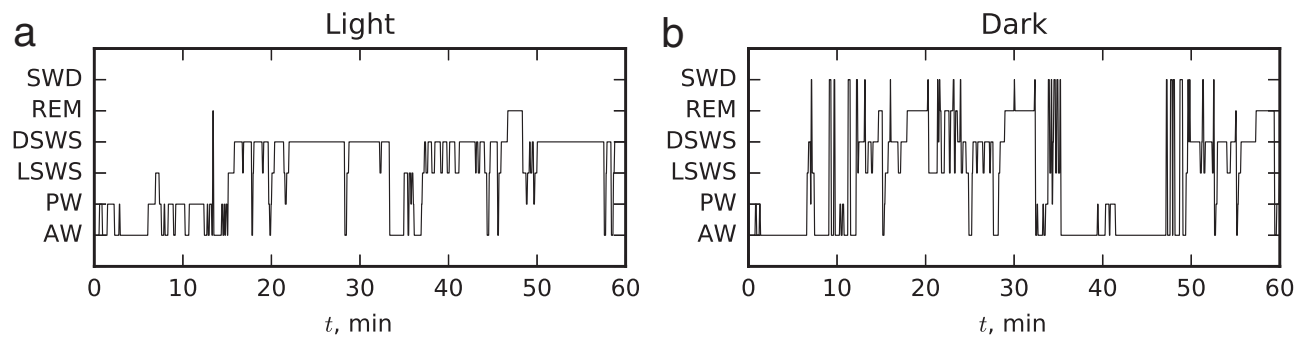

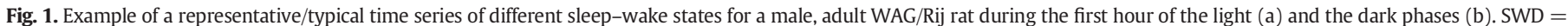
spike-wave discharges, REM = REM sleep, DSWS = deep slow-wave sleep, LSWS = light slow-wave sleep, PW = passive wakefulness, AW = active wakefulness.

of a frequency of $7-11 \mathrm{~Hz}$ (about $10-11 \mathrm{~Hz}$ at the beginning and lowering slightly towards end), duration of at least $1 \mathrm{~s}$, amplitude of at least twice the background EEG, next they were verified visually $[37,38]$. The 2-s epoch length allows to include the putative effects of microarousals and short and quick changes in sleep-wake states on the occurrence of SWDs.

The whole recording was split into continuous, not overlapping 2-s intervals; a number was assigned to each of the considered six states (categories), and this resulted in discrete sequences of values corresponding to the different states. An example of time series of different sleep-wake states is plotted in Fig. 1.

After the procedure of classification, a single continuous time series of categorical (or nominal) data with sampling interval of $2 \mathrm{~s}$ (sampling rate: $0.5 \mathrm{~Hz}$ ) was obtained based on the combined outcomes from the sleep classifier and SWD detection procedure. These time series consist of nominal data: the six states coded with certain numbers/values (it is incorrect to say for example that LSWS is 3 times more than AW, or that AW DSWS = REM) and nominal data do not allow to use cross correlation, coherency, mutual information/transfer entropy, Granger causality etc., to analyze interdependencies between the six states. Moreover, some of these often used methods estimate the interdependencies between time series. Therefore, the main analysis tools used here to analyze a single time series with nominal data were unconditional and conditional probability distributions plotted as histograms; they are still considered as a first analysis step for categorical data [39]. Unconditional distributions were estimated by calculation number of events $p$ $\left(x_{i}\right)$, corresponding to each state, per recording (this value is also known as a "frequency"), and then by normalizing this number to the total number of events. As an event, either any continuous intervals of the considered state $i$ of any length, or 2-s intervals were considered. Conditional distributions were estimated similarly, but only the events of the state $i$ following (or preceding) the chosen state $n$ were considered. Shannon entropy was used as a metric to describe and analyze the probability distributions of the conditional transitions of the six different states in the light and dark phases separately. The conditional transition from state $x_{n}$ was calculated as:

$H\left(x \vee x_{n}\right)_{l}=-\sum_{i=1}^{6} p\left(x_{i} \vee x_{n}\right)_{l} \log \left(p\left(x_{i} \vee x_{n}\right)_{l}\right)$

where $p\left(x_{i} \vee x_{n}\right)_{l}$ is a conditional probability of event $x_{i}$ if the event $x_{n}$ was observed $l$ time points (in our case, each time point equals to $2 \mathrm{~s}$ ) before or will be observed after (if $1<0$ ). For a review of the conditional distribution and Shannon entropy methods, see refs. [40-42].

A chi ${ }^{2}$ test was used to measure the dependency of transitions between categories in the light and dark phases [43-45], followed by $t$-tests to describe differences in percentages of the various states between the light and dark phases.

Finally, it was analyzed whether there is a circadian modulation in the type of transitions to SWD occurrences. Correlations between the hourly number of different transitions and the number of SWDs were determined in every hour of the light-dark (LD) period.

\section{Results}

The $\mathrm{chi}^{2}$ test for categorical data was performed to reveal differences between the distributions of the 6 states in the light and dark phases. This test considers the values plotted in the histograms as presented in Fig. 2, showing the percentage of total time and standardized number of times that an event occurred, respectively. The distribution of the total time occupied by the different states is significantly different for the light and dark periods $(\mathrm{p}<.001)$, however, the distribution of the number of different states shows only a tendency $(\mathrm{p}<.10)$.

Analysis of the percentages of time of the various vigilance states across $24 \mathrm{~h}$ in the light and the dark phases is presented in Fig. 2. It showed the usual phase-related differences regarding total time of AW, LSWS, DSWS, and SWDs (all p-values <.05). Active wakefulness and SWDs predominantly occurred in the dark phase, while DSWS and LSWS preferably were dominantly noticed in the light phase. Rapid eye movement sleep and PW occupied approximately equal amounts of time in each phase.

Comparison between the distribution histograms for the total length and the number of events (Fig. 2) suggested that the mean length of SWDs does not vary between light and dark phases, however, the number of SWDs is much larger in the dark phase. The number of events of AW is relatively small, but their length, especially during the dark phase, is high. Passive wakefulness behaves oppositely: a large number of relatively short episodes. The number of events of LSWS is a bit larger than of DSWS, but deep sleep episodes last longer. While the total time occupied by REM and PW is equal in both phases, the number of events and therefore, their mean length, is slightly different. For PW, it is longer in the light period, for REM sleep longer in the dark period.

\subsection{Conditional distribution}

The conditional distributions of all possible transitions between all 6 states were computed. Since for all considered regimes the mean length of a state is much longer than $2 \mathrm{~s}$, the most frequent transition is always the transition to the same state. Therefore, in order to observe the transitions between different states, not events (i.e., the 2-s epochs), the transition probabilities treating all successive identical events as one state were calculated. Conditional probabilities for the latter approach are plotted in Figs. 3 and 4.

$\mathrm{Chi}^{2}$ tests for categorical data were performed to reveal difference between light and dark. The results show a significant difference for events preceding AW $(\mathrm{p}<.05)$ and LSWS $(\mathrm{p}<.0001)$, and events following LSWS $(\mathrm{p}<.02)$ and DSWS $(\mathrm{p}<.0001)$.

From Figs. 3 and 4, it can be seen that the most frequent event preceding SWDs is AW, while seizures are most often followed by LSWS, $\mathrm{PW}$, and AW. Interestingly, PW is the second most frequent state in the light, and AW in the dark. Active wakefulness is usually preceded or followed by PW, the next most frequent is LSWS in the light phase, but SWDs in the dark phase. After PW, AW most likely occurs, the next most frequent is LSWS in the light phase, and SWDs in the dark 

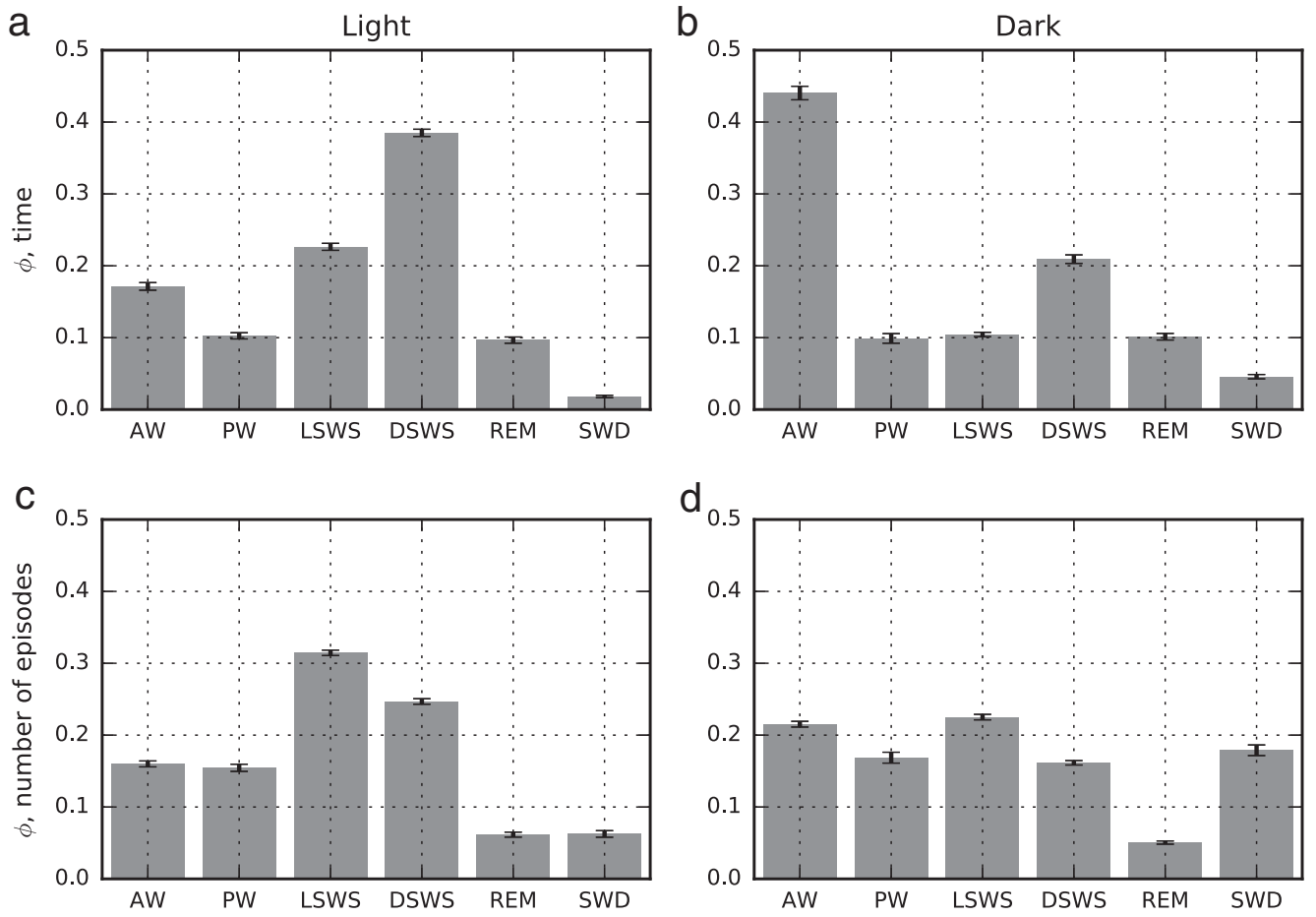

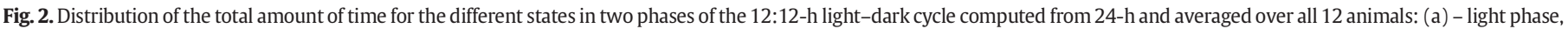

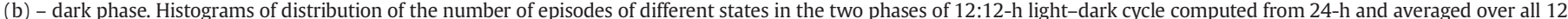

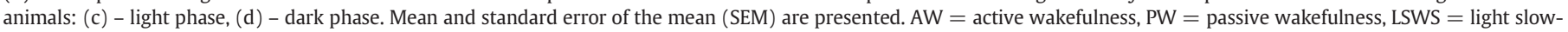
wave sleep, DSWS = deep slow-wave sleep, REM = REM sleep, SWD = spike-wave discharges.

phase. Deep slow-wave sleep switches most likely to LSWS and vice versa in both phases. Second most frequent switch is from PW (albeit with a low probability) in the light phase, but SWDs in the dark. Deep slow-wave sleep is the most frequent state before REM sleep in both the light and dark periods, but the second most frequent is different: LSWS in the light phase and SWDs in the dark phase. Active wakefulness
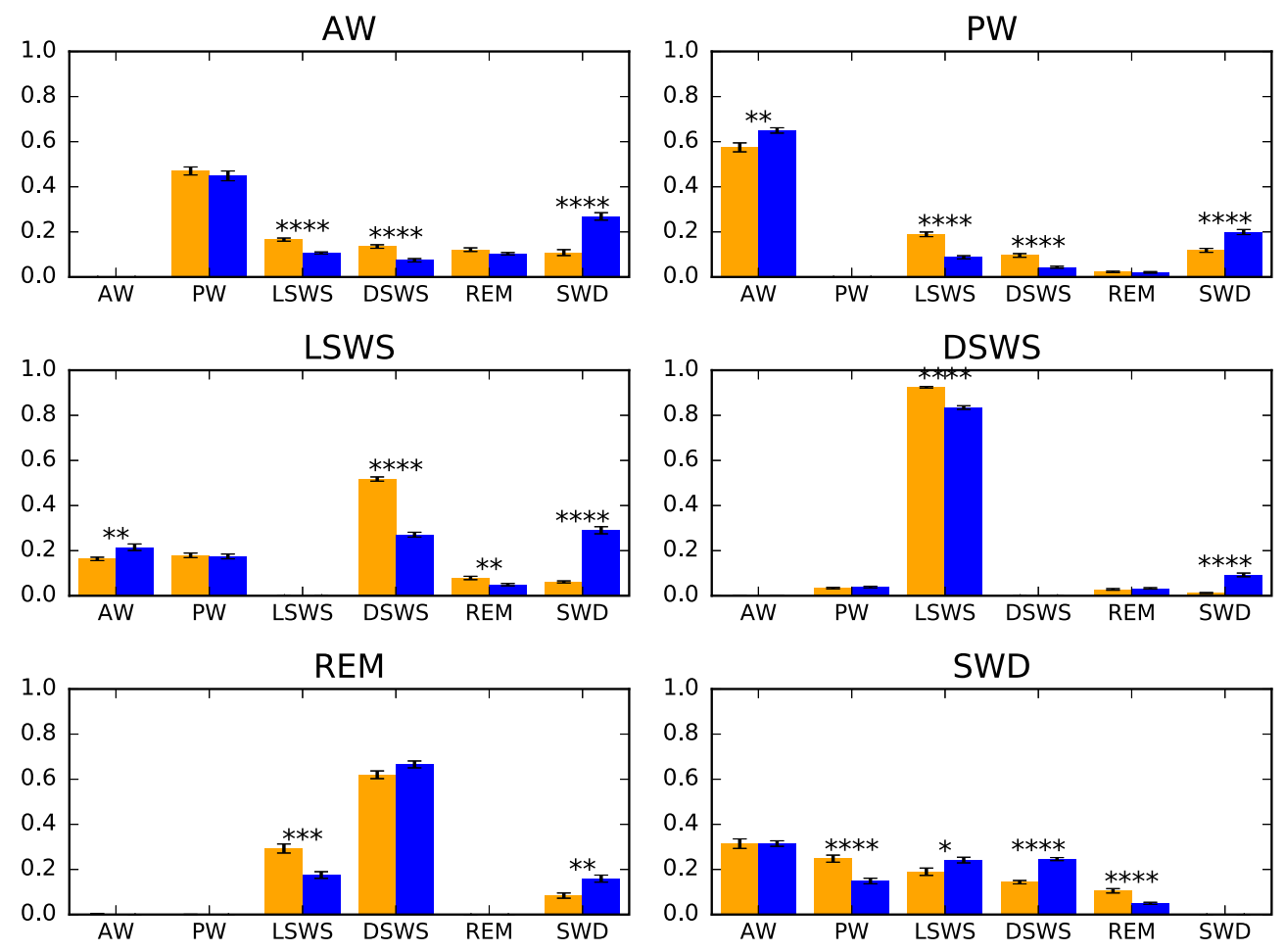

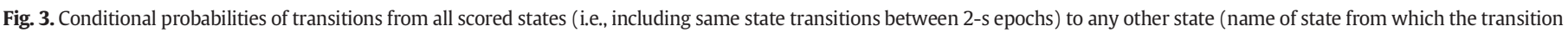

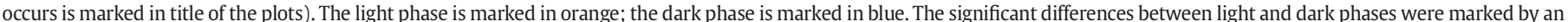

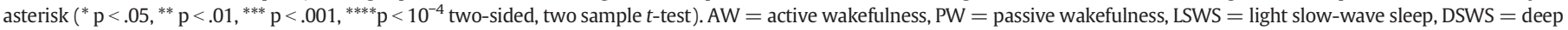

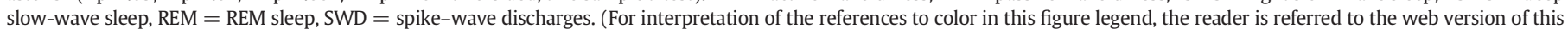
article.) 

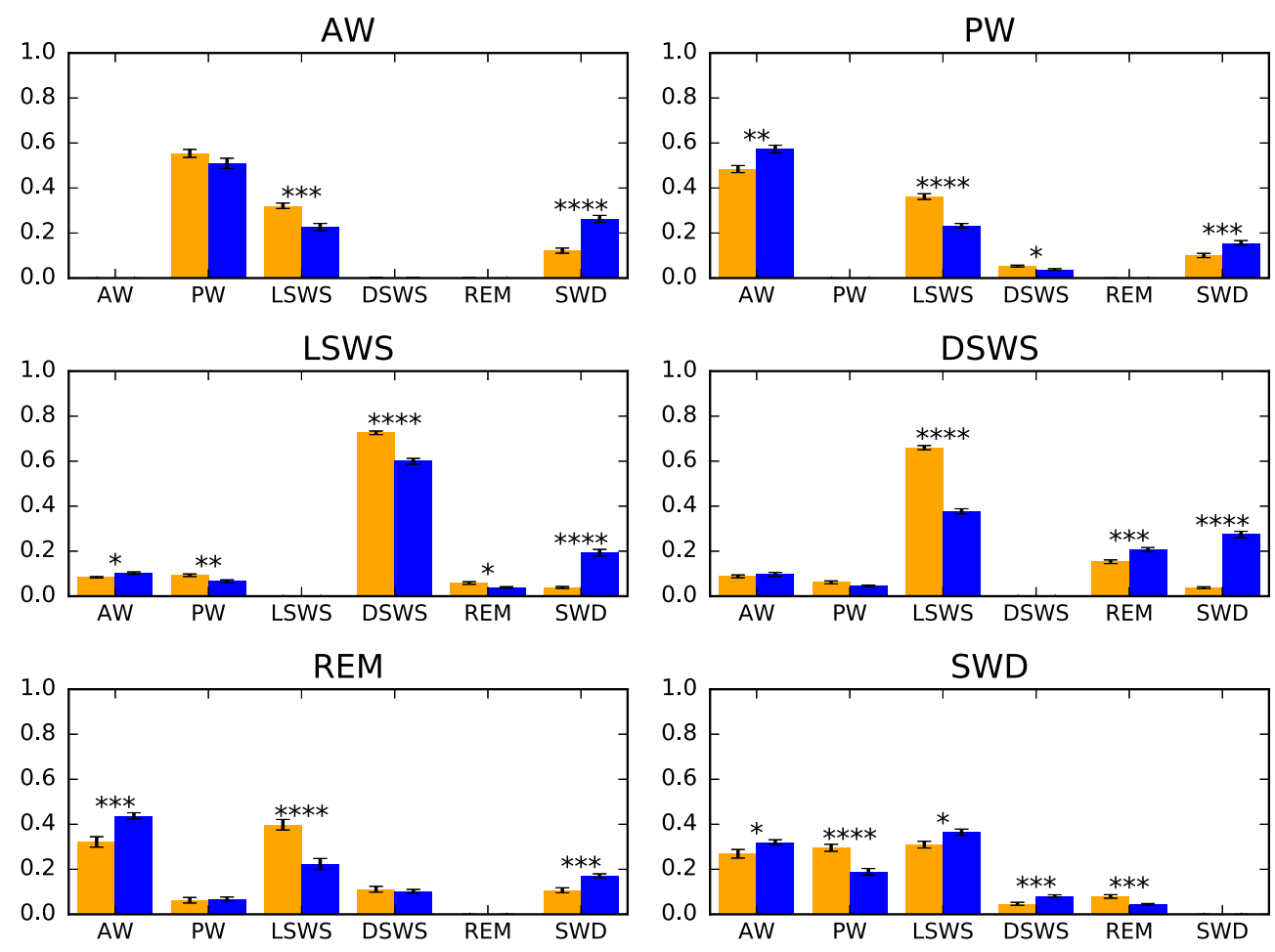

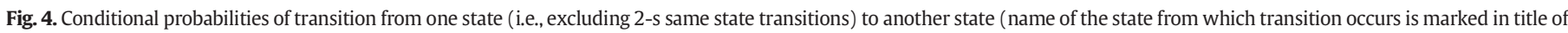

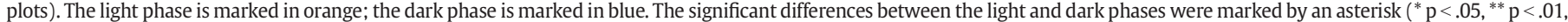

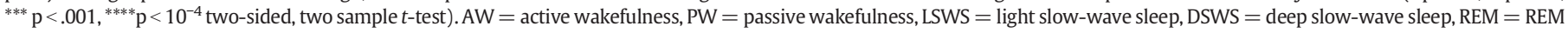
sleep, $\mathrm{SWD}=$ spike-wave discharges. (For interpretation of the references to color in this figure legend, the reader is referred to the web version of this article.)

and LSWS follow REM sleep. Active wakefulness occurs most frequently before SWDs both in the light and dark phases, however, the second most frequent state is different: PW in the light and DSWS in the dark. Spike-wave discharges are mostly followed by L SWS ; PW is the second most frequent in the light, and AW in the dark. To visualize transitions, diagrams of the most frequent transitions are plotted in Fig. 5. The diagram also shows that the SWDs preceding and follow-up states are rather different for the two phases: in the light period, SWDs are mainly preceded by AW and PW and followed by LSWS and PW; in the dark period, SWDs can be preceded by all states and are mainly followed by AW, LSWS, and DSWS (see Figs. 3 and 4 for details).

The analyses of the correlations between the hourly number of different types of transitions and the number of SWDs (data are not presented) showed that the correlations were generally low, but certainly higher in the dark compared to the light phase. The correlations of the number of transitions from AW and DSWS to SWDs and the number of SWDs were higher than for the other three states. Finally, the correlations did not differ a lot in the various hours of the day.

\subsection{Conditional Shannon entropy}

Shannon entropy was calculated from the conditional probabilities of all transition between the states with different time lags $l$ from 2 to $20 \mathrm{~s}$. Shannon entropy helps to integrate results and visualize them, since plotting and analyzing histograms of conditional distributions for many different time lags are time consuming. In addition, Shannon entropy gives a single and comparable value for characterizing the predictability for transitions to and from any state.

The resulting dependences of Shannon entropy for the different lags for all types of states, separated for the light and dark phases, are plotted in Fig. 6. Fig. 6a shows the predictability of a given state based on the previous event type for AW, PW, LSWS, DSWS, REM, and SWDs, while Fig. 6b shows predictability of future events based on the current one. The higher the value of entropy, the lower the predictability of the current type of event (or predictability of event following a current one). $H=0$ corresponds to complete certainty, while the maximal possible value of entropy $\mathrm{H}=\log _{2}(6) \approx 2.585$ corresponds to a completely random process.

Active wakefulness was rather predictable event from the preceding states, its predictability was higher in the dark phase in comparison to the light one (Fig. 6a). Active wakefulness scored also rather high in the prediction of future states and again better in the dark phase of the LD cycle (Fig. 6b). Passive wakefulness is difficult to predict and predicts little, and this was completely independent on the phase of the photoperiod. Deep slow-wave sleep and REM sleep entropy values are also low, therefore, their occurrence can be rather well-predicted, as well as their occurrence predicts other states. Interestingly, the predictability of DSWS and LSWS and how well these two states predict other states is not very different for the light and dark phases, although their predictability is higher in the light than in the dark phase (Fig. 6a and b) and this is opposite to what is found for AW and PW. Deep slow-wave sleep and REM sleep relative flat entropy curves suggest that the presence of either state has consequences for what happens in the subsequent time period. In contrast, LSWS is the least predictable, nor it predicts strongly subsequent states. Rapid eye movement sleep can be better predicted and predicts better other states in the dark phase. Spike-wave discharges have already a high entropy value at the low time lags, the highest of all states and reach the asymptotic value rather quick, more quickly than any other state. This is the case both regarding the states that predict SWDs from the preceding states (Fig. 6a), as well as the predictability of future states based on the presence of SWDs (Fig. 6b). It is interesting that while the number of episodes of SWDs is higher in the dark versus the light phase, its predictability does not differ and remains very small at all time lags except $\mathrm{l}=2 \mathrm{~s}$.

\section{Discussion}

The potential of sleep-wake states for predictability of the occurrence of SWDs across the 24-h light-dark cycle was investigated by 
Light
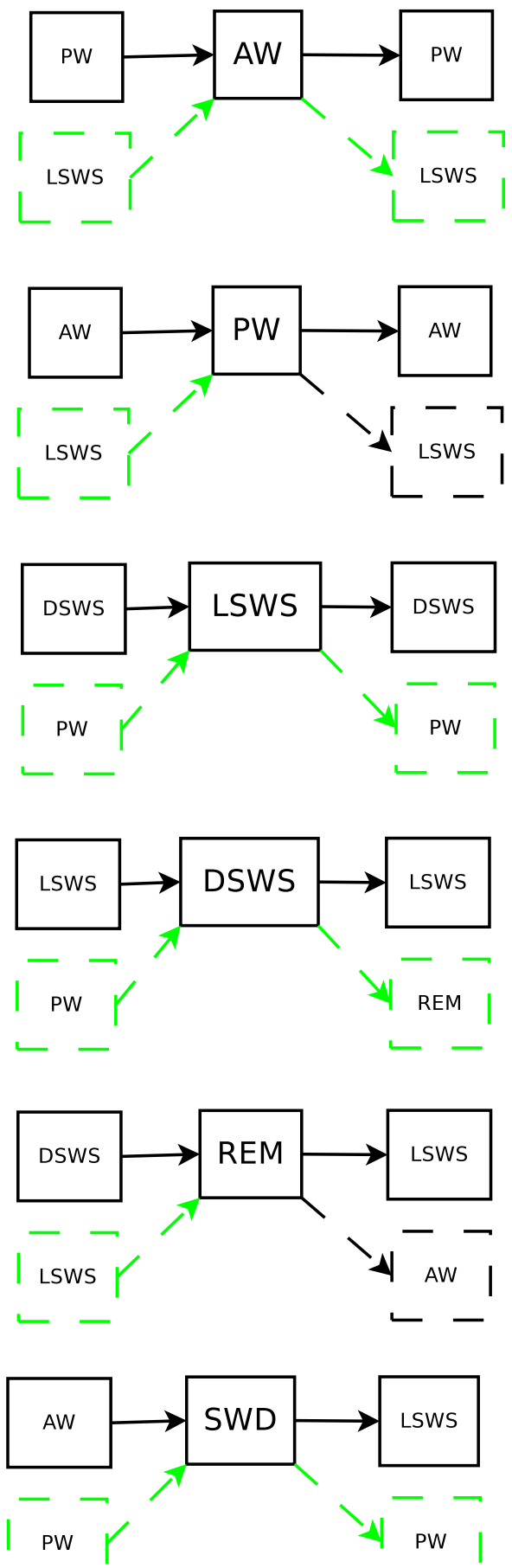

Dark
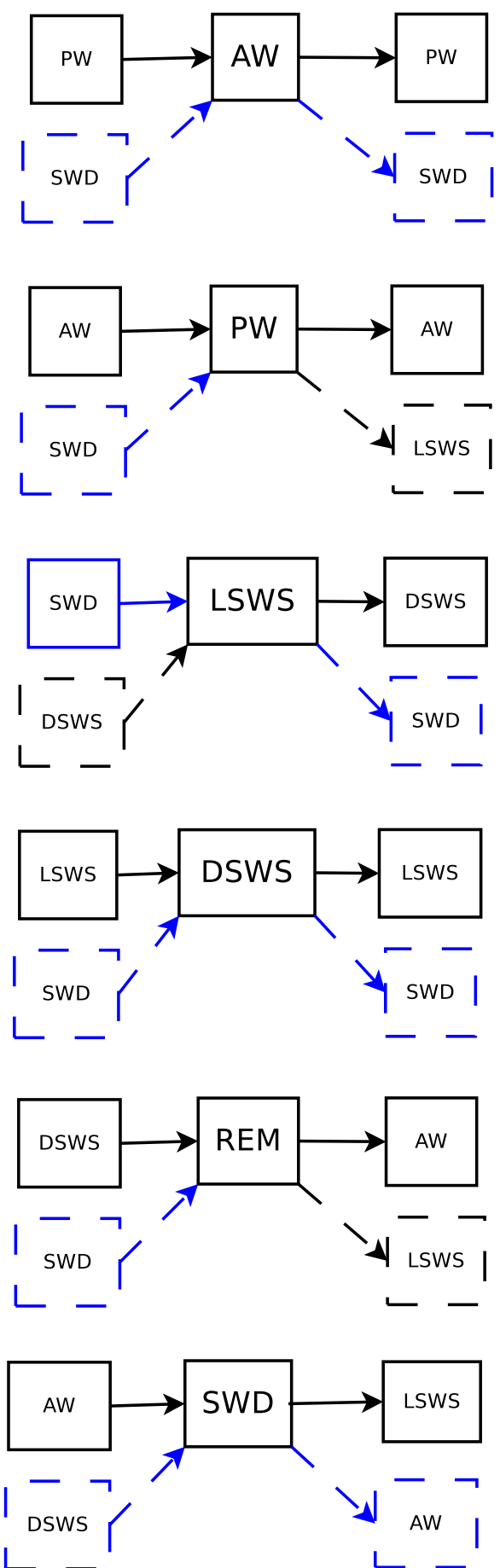

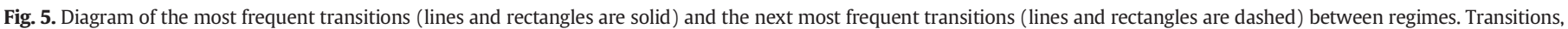

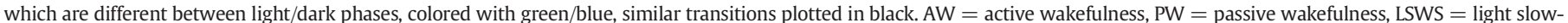

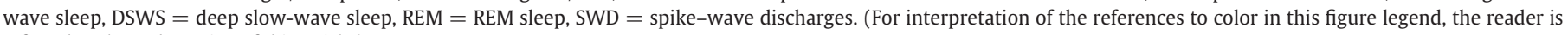
referred to the web version of this article.)

means of conditional probabilities and Shannon entropy in WAG/Rij rats, a well-established genetic animal model of CAE. Significant differences in absolute duration of vigilance states and SWDs, conditional probabilities of transitions between them, and a different level of predictability with regard to the state and the phase of the light-dark cycle have been found.
The study confirmed the well-established phase-related differences in the total duration of sleep-wake states across 24-h cycle in rats [46]. Typically for nocturnal rodents, the dark phase in WAG/Rij rats was dominated by long duration episodes of AW. In the light phase, slowwave sleep, in a form of relatively long bouts of DSWS, predominated. In accordance with previous studies, the number of SWDs prevailed in 

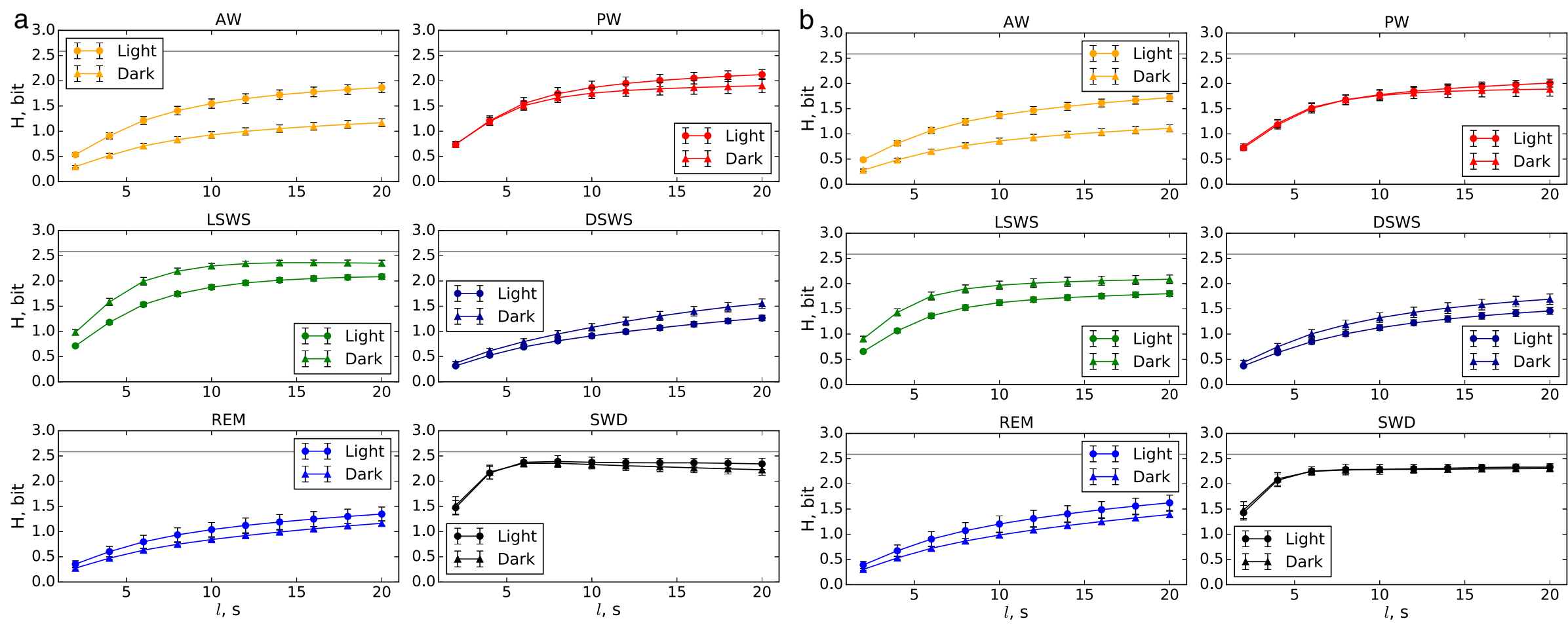

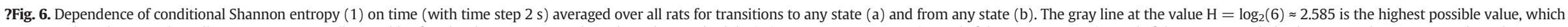

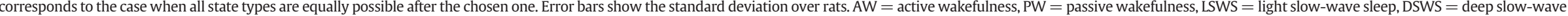
sleep, REM = REM sleep, SWD = spike-wave discharges. 
the dark phase, however, their length did not vary between light and dark [20]. An earlier study investigating the circadian rhythmicity of SWDs in WAG/Rij rats found the same phenomenon: a strong, high amplitude rhythm in the number of SWDs with a clear difference between light and dark. In contrast, the rhythm in the mean duration appeared weak with no phase-related differences $[19,20]$.

Analysis of conditional probability of transitions to and from SWDs revealed that SWDs were most often preceded by AW and followed by LSWS. The relationship was stable regardless of the hour and the phase of the photoperiod, placing SWDs within the pathway from AW, via PW towards LSWS. While in the light phase, SWDs occurred around PW (the second most probable state preceding and following SWDs); in the dark phase, SWDs were seen before all sleep and wake stages and preferentially after both slow-wave sleep stages. Moreover, AW was second to follow SWDs in the dark, suggesting that SWDs were a part of awaking process or the awakenings tend to abort ongoing SWDs. The study of Drinkenburg and coworkers [32], which analyzed the distribution of sleep-wake states preceding and following SWDs in WAG/Rij rats, found PW and LSWS the most favorable states for the occurrence of SWDs. Similar results were obtained in Genetic Absence Epilepsy Rats form Strasbourg (GAERS), in which quiet wake and the beginning of slow-wave sleep were the most frequent to precede SWDs [47]. Active wakefulness directly preceding SWDs was found only in small number of cases, 7 and 9\% in the work of Sysoeva et al. and Drinkenburg et al., respectively [30,32]. These discrepancies between the previous studies and the present work might result from the fact that earlier studies did not include a full 24-h period, but only $4 \mathrm{~h}$ of the dark phase [30] or $6 \mathrm{~h}$ of both phases [32]. Drinkenburg and coworkers analyzed every $5 \mathrm{~s}$ before and after SWDs [32]; in Lannes et al., the EEG was analyzed second by second [47]. In the present study, the resolution of the scoring system was $2 \mathrm{~s}$; the whole $24 \mathrm{~h}$ were analyzed; the phase division was made; and in order to analyze only transitions between different states, all successive events of the same state were treated as one. Accordingly, all the studies, also in patients, reported a transitional period (i.e., in terms of changing from one state of oscillatory brain activity to another) as the SWDs' occurrence zone, however, phaserelated differences in transitions around SWDs as found in the present study, were not shown previously $[6,32,47]$.

Further analysis of the conditional probabilities of the transitions between sleep-wake states (Fig. 7) in the light and dark periods showed that AW was most often followed by PW, and that PW was most often followed by AW. The intertwining of AW and PW in WAG/Rij rats is

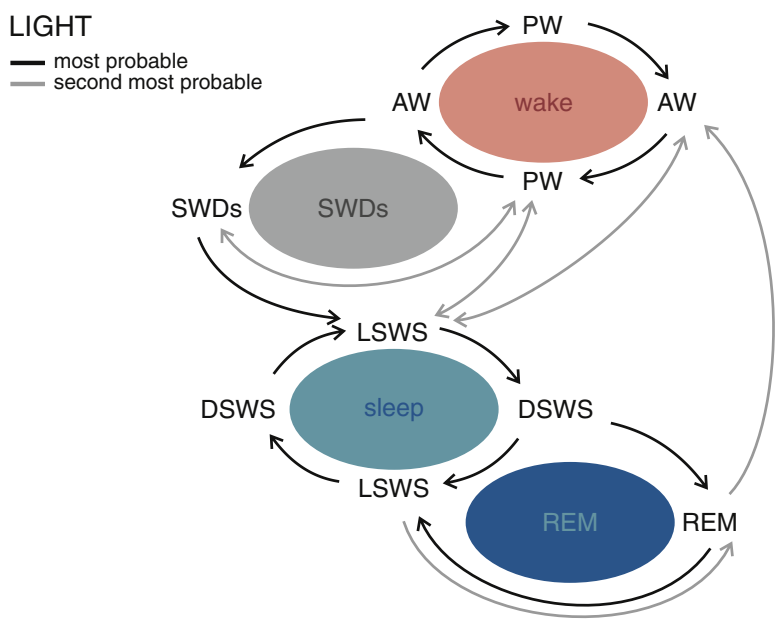

independent of the phase of the photoperiod. The correlation analyses showed little variation between successive hours. These findings suggest a strong and stable bond between these two states; this is in accordance with the study of global forebrain dynamics by Gervasoni et al. [48]. These authors found that the transitions between active and quiet wakefulness are the most frequent among all transitions. A similar relationship was seen for the cycle of slow-wave sleep; here, LSWS was most often followed by DSWS and vice versa, while REM sleep was generally preceded by DSWS, and followed by LSWS in the light and by AW in the dark phase of the 12-12 light-dark phase. However, the close interrelationship was more clearly present during the light phase. In the dark phase, this cycle was interrupted by SWDs being the most probable to precede LSWS and the second most probable to precede DSWS. According to Simasko and Mukherjee [49], the sleep-wake cycle of the rat is characterized by rapid switches between brief periods of wakefulness, slow-wave sleep, and REM sleep. The number of episodes of vigilance cycling was greater in the dark phase, which created more opportunities for sleep-wake transitions, the favorable situation for the occurrence of SWDs. A previous study investigating the sleepwake cycle in WAG/Rij rats revealed that older rats, having more SWDs, had shorter sleep-wake cycle with shorter slow-wave sleep episodes when compared to younger counterparts [50]. Moreover, although in this study only the light phase was investigated, the latter part of it in older rats was characterized by a shorter sleep-wake cycle. Interestingly, none such differences were found in a control nonepileptic strain. Together, these observations suggest that more frequent transitions correlate with increased number of SWDs and a circadian factor modulating the sleep-wake cycle.

Increased frequency of the occurrence of SWDs in the dark phase and the heterogeneity with regard to the sleep-wake states they precede might be also related to the progressive desynchronization of sleep-wake rhythms across $24 \mathrm{~h}$. In rats, entrained to LD 12:12, the sleep-wake cycle is strong and dominated by 24 -h periodicity. However, the circadian factor is not the only determining factor characterizing the occurrence of the different sleep-wake states. Stephenson et al. [51] reported that the period of ultradian rhythms in wake, slow-wave, and REM sleep in rats kept in 12:12 photoperiod varies randomly across a day within a range of 3 to $6 \mathrm{~h}$. Moreover, a phase-related difference was noticed: there was a more prominent rhythm during the light than during the dark phase. This was explained by a progressive desynchronization of ultradian oscillations from the moment of light onset throughout the circadian cycle [51]. Our previous study revealed

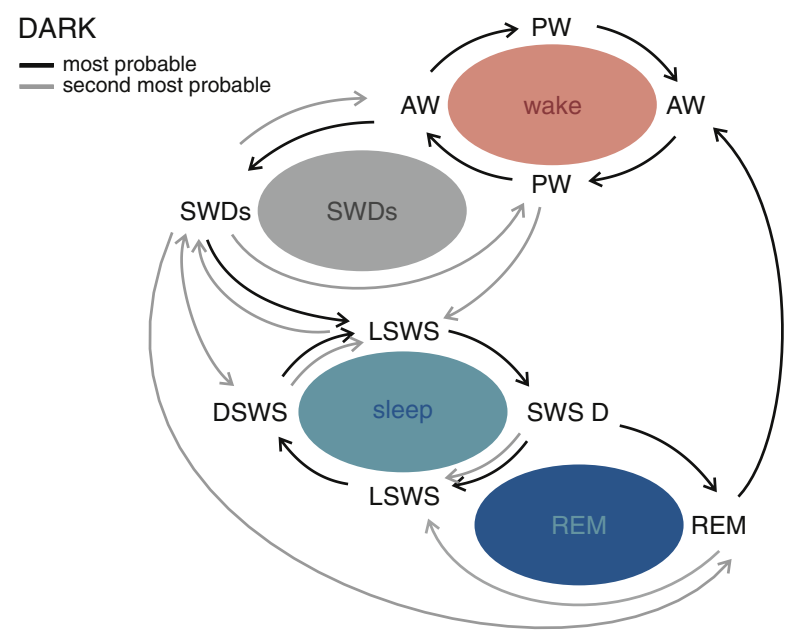

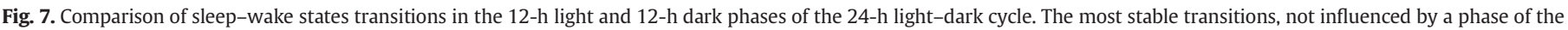

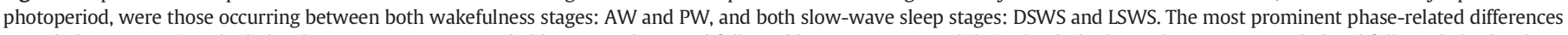

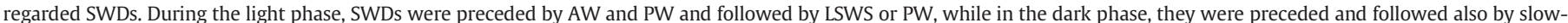

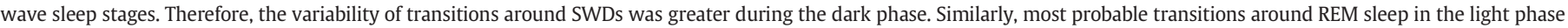

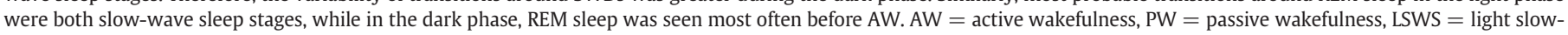
wave sleep, DSWS = deep slow-wave sleep, REM = REM sleep, SWD = spike-wave discharges. 
that circadian desynchronization induced via constant light conditions aggravated epileptic activity (more SWDs) in the same WAG/Rij model [20]. Although incomparable in severity to the complete removal of a potent Zeitgeber, a slight decrease in synchronization between rhythms of different ultradian periods towards the end of the dark period in a 24-h day might contribute to the higher number of SWDs and their preference to occur following different vigilance states.

Another phase-related difference regarded REM sleep. If the rat entered REM sleep in the light phase, it was more probable that the sleep cycle would continue. However, in the dark phase, instead of turning into LSWS, REM sleep was followed by AW more frequently, as reported also by others [52].

Analysis of Shannon entropy revealed that AW was the most predictable, as well as it predicted the other states the best. A phaserelated difference was noted with lower values of Shannon entropy in the dark phase pointing to better predictability in that period. On the other hand, the occurrence of LSWS seems to be difficult to forecast, worse in the dark compared to the light phase. This might be explained by predominance of AW in the dark phase and LSWS in the light phase. However, in other states characterized by phase-related disproportions of total time showing high or very low predictability (DSWS and SWDs, respectively), values of Shannon entropy were not different between phases of the photoperiod. Some other factors than just simply total duration must contribute to phase-related differences in the predictability of specific behavioral states.

Spike-wave discharges were the least predictable events among all states investigated. Their predictability was the highest at time lag $\mathrm{l}=$ $2 \mathrm{~s}$ which can be explained by the fact that the mean duration of SWD is larger than $2 \mathrm{~s}$. At higher time lags entropy quickly increases towards the asymptote (Fig. 6). Spike-wave discharges are strongly modulated by the biological clock and have a strong circadian rhythm, it is obvious that this feature can be used to seizure prediction routines. Otherwise, across the 24-h day, SWDs remain rather "sudden" events on time scales larger of $4 \mathrm{~s}$ and more [22]. This correlates with some recent works, where the earliest changes in network activity preceding SWDs were found at maximum 3-3.3 s before its onset [28,30]. Also, studies investigating the usefulness of permutation and sample entropy for the predictability of SWDs in another commonly used genetic model of absence epilepsy, rats of the GAERS strain, showed a predictability horizon of about 4,9 s and 3,7 s, respectively [53].

Results of the additional hour-by-hour analysis of the correlations between the number of transitions from various sleep-wake states into SWDs and the number of SWDs across 24-h stays in agreement with the results obtained by means of Shannon entropy. The correlations from AW and DSWS transitions with SWDs were more numerous than for the other states; they were also more frequent during the dark phase. Lack of variation in the correlation coefficients across the 24-h period suggests a relative stability of the preference of SWDs to occur after a particular states of vigilance.

The present study investigated the usefulness of the sleep-wake states and the circadian factors for predictability of the occurrence of SWDs. Similar approaches have been used in patients in attempts to refine seizure prediction algorithms. Schelter and coworkers investigated false predictions of seizures, their dependence on the time of a day, and state of vigilance [54]. Adjusting the seizures-prediction method, the dynamic similarity index, by combining different reference states, resulted in almost $50 \%$ reduction in false predictions with only a minor loss of sensitivity [54]. Similarly, incorporation of patient-specific circadian information into electrocorticography-based logistic regression classifiers in the study of implantable seizure-warning device by Karoly and coworkers increased the performance of the seizure-prediction algorithm [55]. Although the present results regarding predictability of SWDs showed that in WAG/Rij rats, SWDs are rather sudden events, detailed investigation of sleep-wake cycle, SWD's relationship with specific states, and circadian factors shaping these relationships, might be combined in already existing SWD prediction algorithms [15].
A final question is why SWDs prefer certain situations within the vigilance continuum. It is well-known that thalamic relay cells fire in two modes: tonic and burst modes. The tonic mode is typical for AW and REM sleep, the burst mode for different types of thalamo-cortical oscillations, either sleep spindles, SWDs, or delta oscillations. The resting membrane potential (RMP) of the thalamic relay and RTN cells is the constituent factor for the type of firing: tonic in case of a depolarized RMP, burst firing when more hyperpolarized. A range of hyperpolarization values determines the frequency of the interspike bursts firing mode; slightly hyperpolarized sleep spindles, SWDs and LSWS, more hyperpolarized, the lower the burst firing frequency and DSWS occurs. Afferents stimuli from the cortex, brainstem, and from the periphery determine the RMP of thalamic relay cells. Excitatory inputs typical for AW and REM sleep depolarize the RMP and thalamo-cortical oscillations of the SWD type are less likely to occur. It is only at the transition phases such as from AW to PW to LSWS that a sufficient number of thalamic cells become slightly hyperpolarized and that the thalamus enters a receptive state to transform the cortical trigger into oscillations of the SWD type. In case of more hyperpolarizations, typical for DSWS, a larger majority of thalamic cells fire with a low interburst frequency reflected by slow delta waves and the thalamus is no longer in a receptive state. Moreover, the cortico-thalamic drive, which may trigger the onset of the generalized SWDs, is reduced during DSWS compared to AW [56]. Evidence, although somewhat indirect, for a particular receptive state is that $8 \mathrm{~Hz}$ after discharges, morphological identical to SWDs and elicited via either cortical or thalamic stimulation, occur mainly during PW and not during AW and DSWS [57,58], and that the thalamic EEG shows a typical low frequency pattern less than $500 \mathrm{msec}$ before the onset of a SWD [26].

\section{Conclusions}

The study assessed whether a distribution of vigilance states organized into 24-h sleep-wake cycle might be useful for the predictability of the occurrence of SWDs in WAG/Rij rats. The results obtained by means of conditional probabilities and Shannon entropy suggest that SWDs possess only a very short prediction possibility (less than $4 \mathrm{~s}$ ): in contrast to deep slow-wave and, to a lesser extend AW. The study revealed also phase-related differences in the organization of the sleepwake cycle and transitions around SWDs: during the light phase, SWDs occurred around PW and participated exclusively in sleep initiation; while in the dark phase, SWDs were seen on both, ascending and descending pathways towards and from sleep. Our results also emphasize, besides the unpredictability of SWDs based on preceding vigilance states, the importance of the circadian factor in the generation of these types of epileptic discharges, and the short prediction window immediately before SWDs onset. These latter characteristics can be implemented into seizure-prediction algorithms increasing prediction rate. The results of the present experiment might find a practical application in better prediction of the previously thought unpredictable generalized seizures.

\section{Conflict of interests}

The authors declare no conflict of interest.

\section{Acknowledgments}

The authors would like to thank Heidi Huysmans and Dirk Nuyts for their excellent technical assistance and express the gratitude to Janssen Research \& Development, a division of Janssen Pharmaceutica NV, Beerse, Belgium for supplying excellent facilities to perform the study.

\section{Funding}

The work was supported by Russian Foundation for Basic Research, Grant 17-02-00307 (the Shannon entropy study), research traveling 
grant from Yuri Gagarin State Technical University of Saratov (international communication necessary for this research), and Stipendium of President of Russian Federation for support of young scientists СП3605.2018.4 (chi-squared test and analysis of unconditional and conditional probabilities).

\section{References}

[1] Seneviratne U, Cook MJ, D'Souza WJ. Electroencephalography in the diagnosis of genetic generalized epilepsy syndromes. Front Neurol 2017;8:499.

[2] Scheffer IE, Berkovic S, Capovilla G, Connolly MB, French J, Guilhoto L, et al. ILAE classification of the epilepsies: position paper of the ILAE Commission for Classification and Terminology. Epilepsia 2017;58:512-21.

[3] Fisher RS, Cross JH, D'Souza C, French JA, Haut SR, Higurashi N, et al. Instruction manual for the ILAE 2017 operational classification of seizure types. Epilepsia 2017;58:531-42.

[4] Steriade M. The corticothalamic system in sleep. Front Biosci 2003;8:d878-99.

[5] Westmijse I, Ossenblok P, Gunning B, van Luijtelaar G. Onset and propagation of spike and slow wave discharges in human absence epilepsy: a MEG study. Epilepsia 2009;50:2538-48.

[6] Halász P, Terzano MG, Parrino L. Spike-wave discharge and the microstructure of sleep-wake continuum in idiopathic generalized epilepsy. Neurophysiol Clin 2002; 32:38-53.

[7] van Luijtelaar EL, van der Werf SJ, Vossen JM, Coenen AM. Arousal, performance and absence seizures in rats. Electroencephalogr Clin Neurophysiol 1991;79:430-4.

[8] Horita H, Uchida E, Maekawa K. Circadian rhythm of regular spike-wave discharges in childhood absence epilepsy. Brain Dev 1991;13:200-2.

[9] Zarowski M, Loddenkemper T, Vendrame M, Alexopoulos AV, Wyllie E, Kothare SV. Circadian distribution and sleep/wake patterns of generalized seizures in children. Epilepsia 2011;52:1076-83.

[10] Seneviratne U, Boston RC, Cook M, D'Souza W. Temporal patterns of epileptiform discharges in genetic generalized epilepsies. Epilepsy Behav 2016;64:18-25.

[11] Halász P, Filakovszky J, Vargha A, Bagdy G. Effect of sleep deprivation on spike-wave discharges in idiopathic generalised epilepsy: a $4 \times 24$-h continuous long term EEG monitoring study. Epilepsy Res 2001;51:123-32.

[12] Roupakiotis SC, Gatzonis SD, Triantafyllou N, Mantouvalos V, Chioni A, Zournas C et al. The usefulness of sleep and sleep deprivation as activating methods in electroencephalographic recording contribution to a long-standing discussion. Seizure 2000;9:580-4.

[13] Ramgopal S, Powell C, Zarowski M, Alexopoulos AV, Kothare SV, Loddenkemper T Predicting diurnal and sleep/wake seizure patterns in paediatric patients of different ages. Epileptic Disord 2014;16:56-66.

[14] Nagaraj V, Lee ST, Krook-Magnuson E, Soltesz I, Benquet P, Irazoqui PP, et al. Future of seizure prediction and intervention: closing the loop. J Clin Neurophysiol 2015; 32:194-206.

[15] Maksimenko VA, van Heukelum S, Makarov VV, Kelderhuis J, Lüttjohann A Koronovskii AA, et al. Absence seizure control by a brain computer interface. Sci Rep 2017;7:2487.

[16] Depaulis A, van Luijtelaar G. Genetic models of absence epilepsy in the rat. In: Pitkänen A, Schwartzkroin PA, Moshe SL, editors. Models of seizures and epilepsy. San Diego: Elsevier Academic Press; 2006. p. 233-48.

[17] Coenen AM, van Luijtelaar EL. The WAG/Rij rat model for absence epilepsy: age and sex factors. Epilepsy Res 1987;1:297-301.

[18] Schridde U, van Luijtelaar G. The role of the environment on the development of spike-wave discharges in two strains of rats. Physiol Behav 2005;84:379-86.

[19] van Luijtelaar ELJM, Coenen AML. Circadian rhythmicity in absence epilepsy in rats. Epilepsy Res 1988;2:331-6.

[20] Smyk MK, Coenen AM, Lewandowski MH, van Luijtelaar G. Endogenous rhythm of absence epilepsy: relationship with general motor activity and sleep-wake states. Epilepsy Res 2011;93:120-7.

[21] van Luijtelaar G, Onat FY, Gallagher MJ. Animal models of absence epilepsies: what do they model and do sex and sex hormones matter? Neurobiol Dis 2014;72:167-79.

[22] Midzyanovskaya I, Strelkov V, van Rij C, Budziszewska B, van Luijtelaar E, Kuznetsova G. Measuring clusters of spontaneous spike-wave discharges in absence epileptic rats. J Neurosci Methods 2006;154:183-9.

[23] Maris E, Bouwman BM, Suffczynski P, van Rijn CM. Starting and stopping mechanisms of absence epileptic seizures are revealed by hazard functions. J Neurosci Methods 2006;152:107-15

[24] Sitnikova E, Hramov AE, Grubov VV, Ovchinnkov AA, Koronovsky AA. On-off intermittency of thalamo-cortical oscillations in the electroencephalogram of rats with genetic predisposition to absence epilepsy. Brain Res 2012;1436:147-56.

[25] Hramov AE, Koronovskii AA, Moskalenko OI, Zhuravlev MO, Jaimes-Reategui R Pisarchik AN. Separation of coexisting dynamical regimes in multistate intermittency based on wavelet spectrum energies in an erbium-doped fiber laser. Phys Rev E 2016;93:052218.

[26] van Luijtelaar G, Hramov A, Sitnikova E, Koronovskii A. Spike-wave discharges in WAG/Rij rats are preceded by delta and theta precursor activity in cortex and thalamus. Clin Neurophysiol 2011;122:687-95.
[27] Sitnikova E, van Luijtelaar G. Cortical and thalamic coherence during spike-wave seizures in WAG/Rij rats. Epilepsy Res 2006;71:159-80.

[28] Lüttjohann A, van Luijtelaar G. The dynamics of cortico-thalamo-cortical interactions at the transition from pre-ictal to ictal LFPs in absence epilepsy. Neurobiol Dis 2012; 47:49-60.

[29] Lüttjohann A, van Luijtelaar G. Dynamics of networks during absence seizure's onand offset in rodents and man. Front Physiol 2015;5:6-16.

[30] Sysoeva MV, Lüttjohann A, van Luijtelaar G, Sysoev IV. Dynamics of directional coupling underlying spike-wave discharges. Neuroscience 2016;314:75-89.

[31] Coenen AM, Drinkenburg WH, Peeters BW, Vossen JM, van Luijtelaar EL. Absence epilepsy and the level of vigilance in rats of the WAG/Rij strain. Neurosci Biobehav Rev 1991:15:259-63.

[32] Drinkenburg WH, Coenen AM, Vossen JM, Van Luijtelaar EL. Spike-wave discharges and sleep-wake states in rats with absence epilepsy. Epilepsy Res 1991;9:218-24.

[33] Paxinos G, Watson C. The rat brain in stereotaxic coordinates. San Diego: Academic Press; 1998.

[34] Ahnaou A, Drinkenburg WH. Neuromedin U(2) receptor signaling mediates alteration of sleep-wake architecture in rats. Neuropeptides 2011;45:165-74.

[35] Lopes da Silva F. EEG analysis: theory and practice. In: Schomer DL, Lopes da Silva F, editors. Niedermeyer's electroencephalography: basic principles, clinical applications, and related fields. Lippincott Williams \& Wilkins; 2012. p. 1147-77.

[36] Corsi-Cabrera M, Guevara MA, Del Río-Portilla Y, Arce C, Villanueva-Hernández Y. EEG bands during wakefulness, slow-wave and paradoxical sleep as a result of principal component analysis in man. Sleep 2000;23:738-44.

[37] van Luijtelaar EL, Coenen AM. Two types of electrocortical paroxysms in an inbred strain of rats. Neurosci Lett 1986;70:393-7.

[38] Ovchinnikov A, Lüttjohann A, Hramov A, van Luijtelaar G. An algorithm for real-time detection of spike-wave discharges in rodents. J Neurosci Methods 2010;194:172-8.

[39] Agresti A. An introduction to categorical data analysis. . 2nd ed.Hoboken, USA: Wiley-Interscience; 2007.

[40] Gnedenko BV. Theory of probability. . 6th ed.CRC Press; 1998.

[41] Klimontovich YL. Entropy and information of open systems. Uspekhi-Physics 1999; 42:375-84.

[42] Gray MR. Entropy and information theory. . 2nd ed.Springer; 2011.

[43] Jaeger F. Categorical data analysis: away from ANOVAs (transformation or not) and towards logit mixed models. J Mem Lang 2008;59:434-46.

[44] Margolin BH, Light RJ. An analysis of variance for categorical data, II: small sample comparisons with chi square and other competitors. J Am Stat Assoc 1974;69: 755-64.

[45] Rao JNK, Scott AJ. The analysis of categorical data from complex sample surveys: chisquared tests for goodness of fit and independence in two-way tables. J Am Stat Assoc 1981;76:221-30.

[46] Borbely AA, Neuhaus HU. Daily pattern of sleep, motor activity and feeding in the rat: effects of regular and gradually extended photoperiods. J Comp Physiol 1978; 124:1-14.

[47] Lannes B, Micheletti G, Vergnes M, Marescaux C, Depaulis A, Warter JM. Relationship between spike-wave discharges and vigilance levels in rats with spontaneous petit mal-like epilepsy. Neurosci Lett 1988;94:187-91.

[48] Gervasoni D, Lin SC, Ribeiro S, Soares ES, Pantoja J, Nicolelis MA. Global forebrain dynamics predict rat behavioral states and their transitions. J Neurosci 2004;24: 11137-47.

[49] Simasko SM, Mukherjee S. Novel analysis of sleep patterns in rats separates periods of vigilance cycling from long-duration wake events. Behav Brain Res 2009;196: 228-36.

[50] van Luijtelaar G, Bikbaev A. Midfrequency cortico-thalamic oscillations and the sleep cycle: genetic, time of day and age effects. Epilepsy Res 2007;73:259-65.

[51] Stephenson R, Lim J, Famina S, Caron AM, Dowse HB. Sleep-wake behavior in the rat: ultradian rhythms in a light-dark cycle and continuous bright light. J Biol Rhythms 2012;27:490-501.

[52] Stephenson R, Famina S, Caron AM, Lim J. Statistical properties of sleep-wake behavior in the rat and their relation to circadian and ultradian phases. Sleep 2013;36: 1377-90.

[53] Li X, Ouyang G, Richards DA. Predictability analysis of absence seizures with permutation entropy. Epilepsy Res 2007;77:70-4

[54] Schelter B, Winterhalder M, Maiwald T, Brandt A, Schad A, Timmer J, et al. Do false predictions of seizures depend on the state of vigilance? A report from two seizure-prediction methods and proposed remedies. Epilepsia 2006;47:2058-70.

[55] Karoly PJ, Ung H, Grayden DB, Kuhlmann L, Leyde K, Cook MJ, et al. The circadian profile of epilepsy improves seizure forecasting. Brain 2017;140:2169-82.

[56] Zobeiri M, van Luijtelaar G, Budde T, Sysoev IV. The brain network in a model of thalamocortical dysrhythmia. Brain Connect 2019. https://doi.org/10.1089/brain. 2018.0621.

[57] Lüttjohann A, Zhang S, de Peijper R, van Luijtelaar G. Electrical stimulation of the epileptic focus in absence epileptic WAG/Rij rats: assessment of local and network excitability. Neuroscience 2011;188:125-34. https://doi.org/10.1016/j.neuroscience.2011.04.038.

[58] Lüttjohann A, van Luijtelaar G. Thalamic stimulation in absence epilepsy. Epilepsy Res 2013;106(1-2):136-45. https://doi.org/10.1016/j.eplepsyres.2013.03.009. 